\title{
Temporal and Spatial Correlations in Electron- Induced Arcs of Adjacent Dielectric Islands
}

\author{
Justin Christensen, JR Dennison, Member, IEEE, and Justin Dekany
}

\begin{abstract}
This study investigates very short duration ( $<\mathbf{1 m s )}$ flashes caused by rapid discharge arcs from isolated, charged, insulating epoxy "glue dots" to an underlying grounded substrate while under electron bombardment. The possibility that a given arc might stimulate arcs in adjacent "glue dots" was investigated through coincidence correlation analysis, as was the dependence of such correlations with "glue dot" separation. Most arcs were found to be random localized events, which occurred only when accumulated charge produced an electric field large enough for electrostatic breakdown to occur. However, for $40 \mathrm{keV}$ incident beams, significant temporal and spatial correlation were observed. It is hypothesised that at higher energies more samples are charged close to the breakdown field at any given time and that a discharge in one "glue dot" might cause a sudden electric field spike in neighbouring "glue dots", which could trigger premature arcing. Such stimulated arc rates might reasonably be expected to scale with electric field intensity. A power law fit to the arc data found a power of $-1.06 \pm 0.09$, consistent with a field falling off inversely with separation distance for charges spreading out across a $2 D$ conducting surface.
\end{abstract}

Index Terms - arc discharge, dielectric breakdown, dielectric materials, electrostatic discharges, materials testing, light emission, space environment effects

\section{INTRODUCTION}

$\mathrm{D}$ ielectric materials exposed to energetic electron fluxes similar to those in space plasma environments can emit light in various forms [1]. It is important to understand these charge and discharge phenomena that occur under space-like conditions, because spacecraft charging is the leading environmental cause of spacecraft anomalies [2,3]. Two forms of electron-induced light emission have been commonly encountered, both of which emit light from large surface areas that have been charged by the incident electron flux $[4,5]$. Continuous emission observed whenever a material is exposed to electron fluxes is termed "glow," or more properly, cathodoluminescence (CL) [4,6-8]. Intermediate-duration light emissions events, which start with a large rapid spike in intensity similar to arcs and are followed by an exponential decay ( 10-100 s decay constant) back to the continuous equilibrium CL intensity, have also been observed for several polymeric and composite materials $[1,5]$.

This study examined an even more common form of electron-induced light emission, short-duration ( $<1 \mathrm{~ms})$, bright

This work was supported by funding through NASA Goddard Space Flight Center and the James Webb Space Telescope.

Justin Christensen, JR Dennison, and Justin Dekany are with the Materials Physics Group in the Physics Department at Utah State University in Logan, photon emissions termed "arcs," which are caused by the rapid discharge of charged insulators [9]. These arcs are often, but not always, localized to smaller regions of the charged surface. In previous studies of spacecraft charging, arcing sometimes occurs in neighboring samples nearly simultaneously even though they are electrically isolated from one another. In this study we investigated the relationship between coincident arcing events of nearby charged samples and sample separation.

The original focus of this study was on spacecraft charging applications, with dielectric samples on mm length scales, insulator spacings on $\mathrm{cm}$ length scales, and surface voltages on $\mathrm{kV}$ scales. However, the physical processes involved have farreaching applications with similar electric fields related to electrostatic breakdown field strength and arcing. Theses include-in order of decreasing length scales-high voltage devices and switching circuits, insulators in plasma applications, breakdown field measurements [2,10], microelectronics capacitors and dielectric layers, and nanodielectric composites [11].

\section{EXPERIMENTAL METHODS}

\section{A. Samples}

The samples studied were numerous separate insulators on a large conductive substrate (see Fig. 1). The insulators were 36 separate, electrically-isolated, small $(\sim 3 \mathrm{~mm}$ diameter), approximately hemispherical, bisphenol/amine epoxy "glue dots" (see Fig. 2). These were located around the edges of a large, conductive, polymeric/carbon nanocomposite (Black Kapton $^{\mathrm{TM}}$ ) substrate (see Fig. 1) [11]. The substrate was mounted inside a high-vacuum chamber, attached to a cooled, grounded, metal plate. The "glue dot" numbering scheme, used in the analysis sections below, is shown in Fig. 1; it begins at the top left, continues clockwise around the periphery of the sample substrate, and finishes with the four "glue dots" in the lower right corner.

\section{B. Instrumentation}

The data collected for this study were taken at NASA Marshall Space Flight Center using a high-vacuum chamber $\left(<10^{-4} \mathrm{~Pa}\right)$. The sample was cooled to $\sim 120 \mathrm{~K}$ using liquid nitrogen. The chamber was equipped with a monoenergetic, high-energy (12-40 keV) electron flood gun to simulate electron fluxes and energies seen in a typical space environment. The glue dots were exposed simultaneously to

UT 84322 USA (e-mail: Justin.Christensen@aggiemail.usu.edu, JR.Dennison@usu.edu, JDekany.phyx@gmail.com ).

Color versions of one or more figures in this paper are available online at http://ieeexplore.ieee.org.

Digital object identifier. 

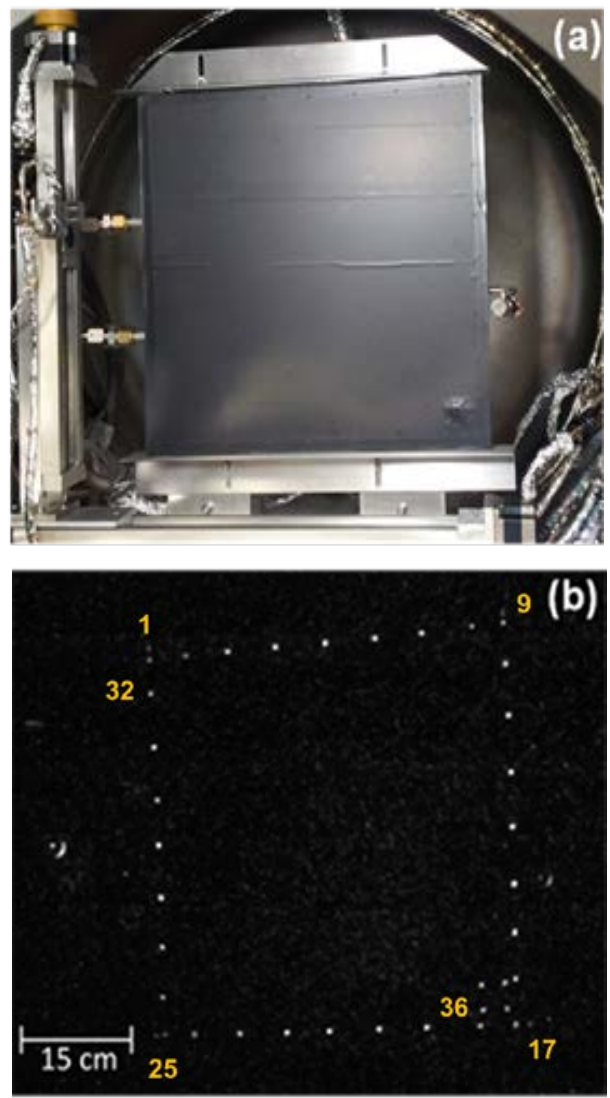

Fig. 1. Sample tested. (a) $41 \times 41 \mathrm{~cm}$ conductive plate mounted in the vacuum chamber. Liquid nitrogen feed lines are visible at the left of the sample. A Faraday cup is seen at right. (b) Sample in dark chamber under electron beam irradiation showing 36 "glue dots" luminescing around the periphery of the sample substrate. The "glue dot" numbering scheme is shown in yellow.

nearly identical electron fluxes $\left(0.3-5 \mathrm{nA}-\mathrm{cm}^{-2}\right)$. Beam uniformity was measured to be $\pm 5 \%$, using a movable Faraday cup mounted next to the sample plate; this consistent exposure allowed for better stochastic study of the various types of light emissions.

Light emitted from the samples was monitored using a visible to near IR (400-900 nm), high-sensitivity, image-intensified CCD video camera (Xybion, ITT ISG-780-1180) at a frame rate of 30 frames per second. This camera was calibrated using an NIST traceable blackbody light source with a known intensity at given wavelengths to determine a calibration factor; this was used to convert from pixel intensity counts to absolute spectral radiance values [4]. For bisphenol/amine epoxy, the best estimates for cathodoluminescence material properties are a spectral radiance per incident power density of $(1.98 \pm 0.04) \cdot 10^{-}$ ${ }^{9}\left[\mathrm{~W}-\mathrm{cm}^{-2}-\mathrm{nm}^{-1}-\mathrm{sr}^{-1}\right.$ per $\left.\mu \mathrm{W}-\mathrm{cm}^{-2}\right]$, a saturation dose rate of 420 $\mu \mathrm{W}-\mathrm{cm}^{-2}( \pm 30 \%)$, and a saturation/de-saturation time constant of $120 \pm 40 \mathrm{~s}$ [4].

\section{Data Acquisitions}

In order to analyse the data, some video frames with a light on the samples were used to define locations for each "glue dot" [see Fig. 2(b)]. A series of dark video frames were also acquired immediately prior to turning the electron beam on, in order to get a good baseline for background subtraction to

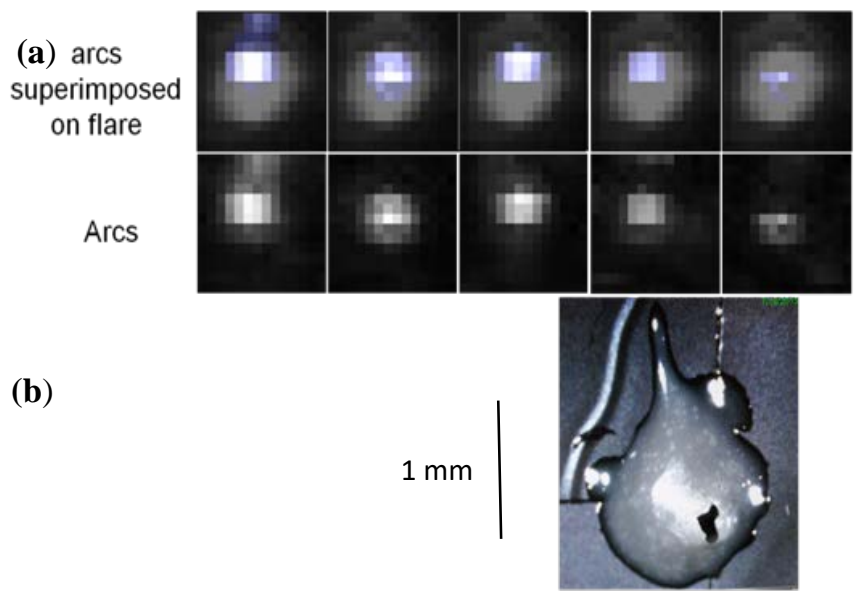

Fig. 2. (a) Identical regions of various pixilated video frames, to show the variations in spatial locations of arcs around the "glue dot" for different arcs on a particular glue dot. Arc images are also shown superimposed on images of the "glue dot" when it is illuminated by a flare in the background. (b) Higher magnification image of the "glue dot" under brighter illumination can be used to reference approximate arc locations.

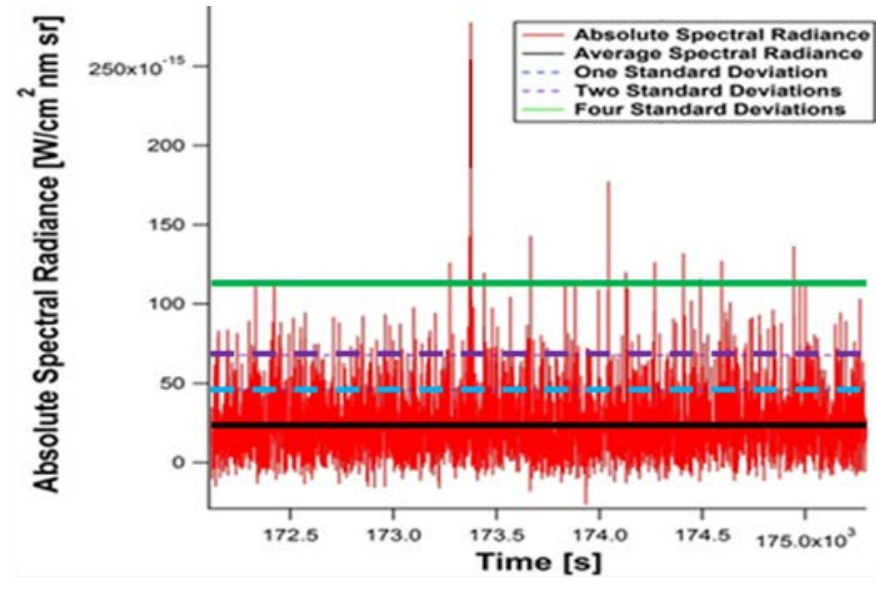

Fig. 3. Typical curve of average corrected absolute spectral radiance versus exposure time for a single "glue dot". The average spectral radiance and levels at 1,2 , and 4 standard deviations above this are indicated. An average background has been subtracted to account for stray light contamination.

remove noise and stray light contamination from ambient lights and the electron gun filament glow.

The samples were exposed to the electron fluxes for about 15 min at each energy, with a 2 min break in between each energy. While these light emission data were being acquired, current from the large conductive plate was monitored; the plate current was used as a secondary standard after having been calibrated against Faraday cup measurements of the absolute incident current density.

\section{DATA ANALYSIS}

\section{A. Video Processing}

The video files were converted to individual .jpg images and analyzed with a custom Matlab program designed for this study. This program allowed for various regions of the substrate images with different shaped areas to be selected for analysis by the user. For each individual video frame, the pixel intensity values for all pixels in each region were summed, divided by 
the number of pixels, and multiplied by a calibration factor for the video camera to determine the average absolute spectral radiance emitted from that region in each frame. Average absolute spectral radiance values for several background regions were also determined with the electron gun on but with the beam blanked. These were used to perform background subtraction for stray light contamination.

An array of calibrated intensities (average corrected absolute spectral radiances) versus time for sequential frames was calculated separately for regions associated with each glue dot. Fig. 3 shows a typical curve of corrected absolute spectral radiance versus exposure time for a single "glue dot". Note that subtraction of an average background led to a small fraction ( $\sim 4 \%$ ) of the corrected absolute spectral radiance values with unphysical values less than zero (see Figs. 3 and 4); however, this did not affect the subsequent analysis to identify arc events discussed in the next section.

\section{B. Arcing Analysis}

An algorithm was developed to determine if an arc occurred in each frame of the luminescence data. Several methods were explored to determine the most accurate way to identify a current threshold in curves of absolute spectral radiance versus exposure time for a single "glue dot" (e.g., Fig. 3); any data point with intensity above the threshold was deemed an arc. The
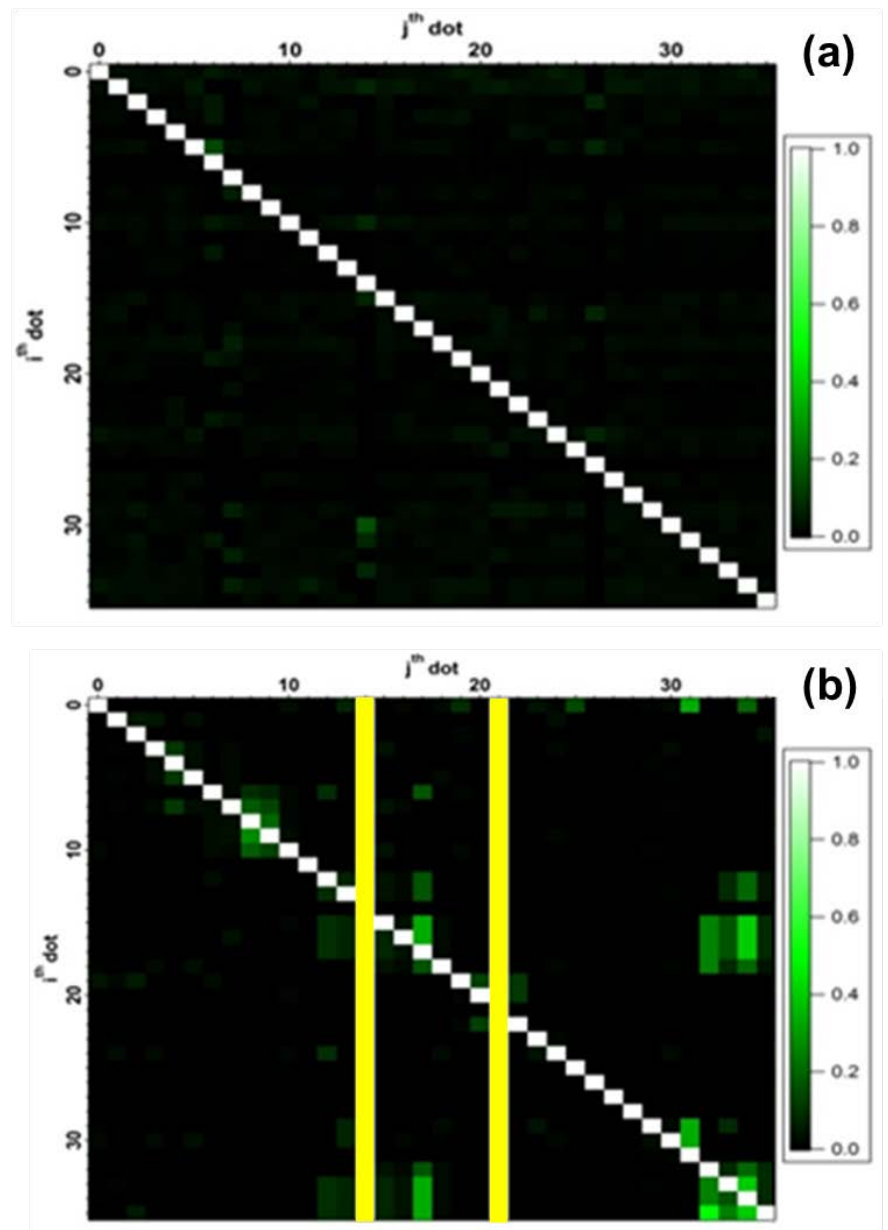

Fig. 5. Correlation matrices for "glue dot" arcs, as determined with Eq. (1). Correlation values for incident electron energies (a) $25 \mathrm{keV}$ and (b) $40 \mathrm{keV}$.

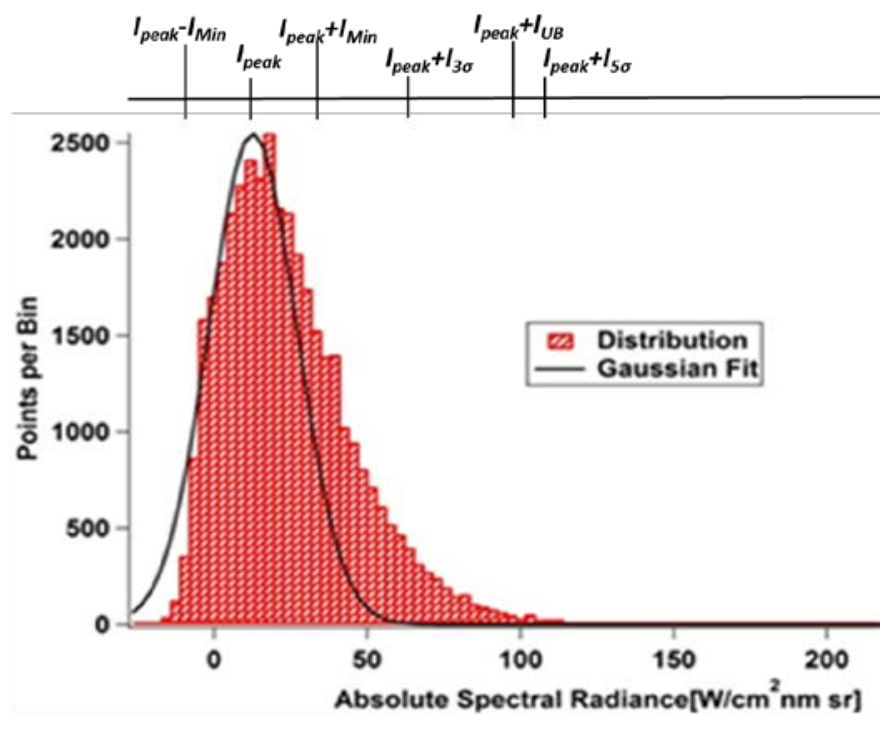

Fig. 4. Histogram of corrected absolute spectral radiance for a single analysis region over prolonged beam exposure. An average background has been subtracted to account for stray light contamination. Comparison to a Gaussian fit shows the intensity distribution is decidedly asymmetric. Various threshold values used to identify arcs are shown above the histogram.

arc threshold was variously set (see Fig. 4) as:
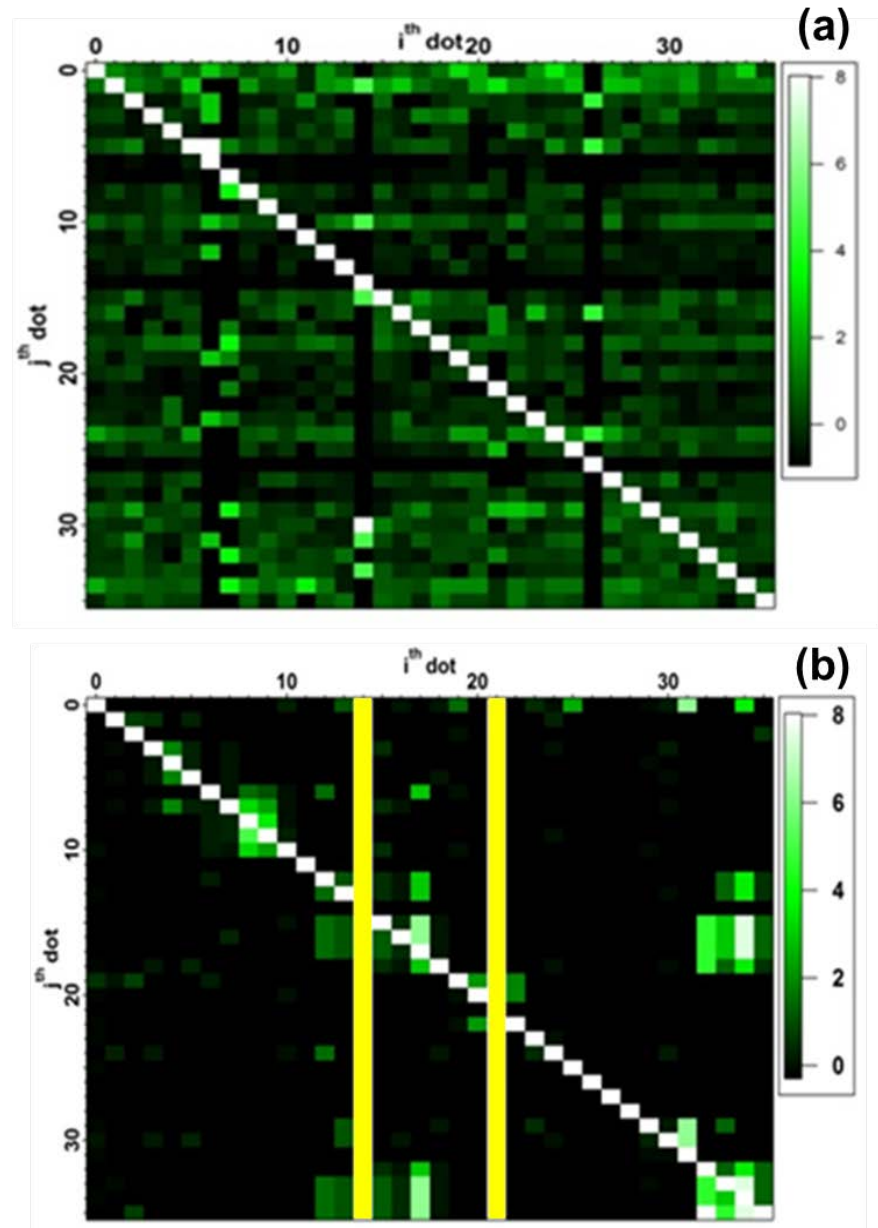

Fig. 6. Normalized correlation matrices for "glue dot" arcs, as determined with Eq. (2). Correlation values for incident electron energies (a) $25 \mathrm{keV}$ and (b) 40 $\mathrm{keV}$. 
(i) the peak spectral radiance, $I_{\text {peak }}$, plus $1 \sigma, 2 \sigma, 3 \sigma, 4 \sigma$, or $5 \sigma$ denoted $I_{l \sigma}, I_{2 \sigma}, I_{3 \sigma}, I_{4 \sigma}$, and $I_{5 \sigma}$,

(ii) the peak spectral radiance plus a similar factor of 1 to 5 times the range of spectral radiance calculated as the average minus the minimum non-zero intensity, denoted $I_{\text {Min } 1}, I_{\text {Min } 2}, I_{\text {Min } 3}, I_{\text {Min } 4}$ and $I_{\text {Min5 }}$, or

(iii) the upper bound (first zero value to the right of the peak) of a histogram of the spectral radiance data, denoted $I_{U B}$.

The various methods used to determine the intensity threshold to define arcs produced similar values for the relative arc rates. Average aggregate arc rates of 0.5 to 2 arcs per min for all "glue dots" were observed, with the rate exhibiting a small increase with increasing incident energy [4]. Upon inspection of the video frames to manually look for arcs, it became evident that arcs varied greatly in intensity as well as spatial extent. This most likely caused many arcs to be lost in the noise, and it may account for the asymmetry of the intensity distributions observed (Fig. 4). In the end, the most reliable method for counting arcs was found to be creation of a histogram of the intensities and to define the threshold as the upper bound of the distribution, $I_{U B}$.

\section{Correlation Analysis}

A temporally correlated—or coincidence-arc was defined to be an arc that occurred within \pm 1 frame ( $\pm 33 \mathrm{~ms}$ ) of an arc in a separate sample. To test for spatial correlation between coincidence arcs in nearby samples, the following definition was used to calculate the fraction of arcs in sample $j$ temporally correlated with arcs in sample $i$. The total number of arcs in sample $j$ coincident with arcs in sample $i, N_{i, j}$, was divided by the total number of arcs in sample $i, N_{\text {total }}(i)$ to determine a correlation value between samples $i$ and $j$ for the element $C_{i, j}$ :

$$
C_{i, j} \equiv N_{i, j} / N_{\text {total }}(i)
$$

This produced a two dimensional correlation matrix with values ranging from 0 (no correlation between the samples $i$ and $j$ ) to 1 (perfect correlation). In principle, the correlation matrix should be symmetric about the diagonal with $C_{i, j}=C_{j, i}$, In practice, the correlation matrix is not fully symmetric due to uncertainties inherent in low count rates, such that $N_{\text {total }}(i) \neq$ $N_{\text {total }}(i)$.

Figures 5(a) and 5(b) show two examples of this analysis for $25 \mathrm{keV}$ and $40 \mathrm{keV}$ incident energies. Dark areas have low correlations and green areas show higher correlations between pairs of "glue dots". The white diagonal elements are perfect self-correlations. Yellow vertical stripes in Figs. 5(b) and 6(b) indicate pixels that had no arcing at $40 \mathrm{keV}$.

To improve the contrast of the non-diagonal elements of the correlation matrix, their values were normalized to show how each element related to the average, $C_{\text {avg }}$, and standard deviation, $\sigma_{C}$, of the correlation matrix:

$$
C_{i, j}^{\text {norm }}=\left(C_{i, j}-C_{a v g}\right) / \sigma_{C}
$$

Thus, for example, a normalized correlation matrix value of 8 indicates a coincidence rate between sample pairs 8 standard
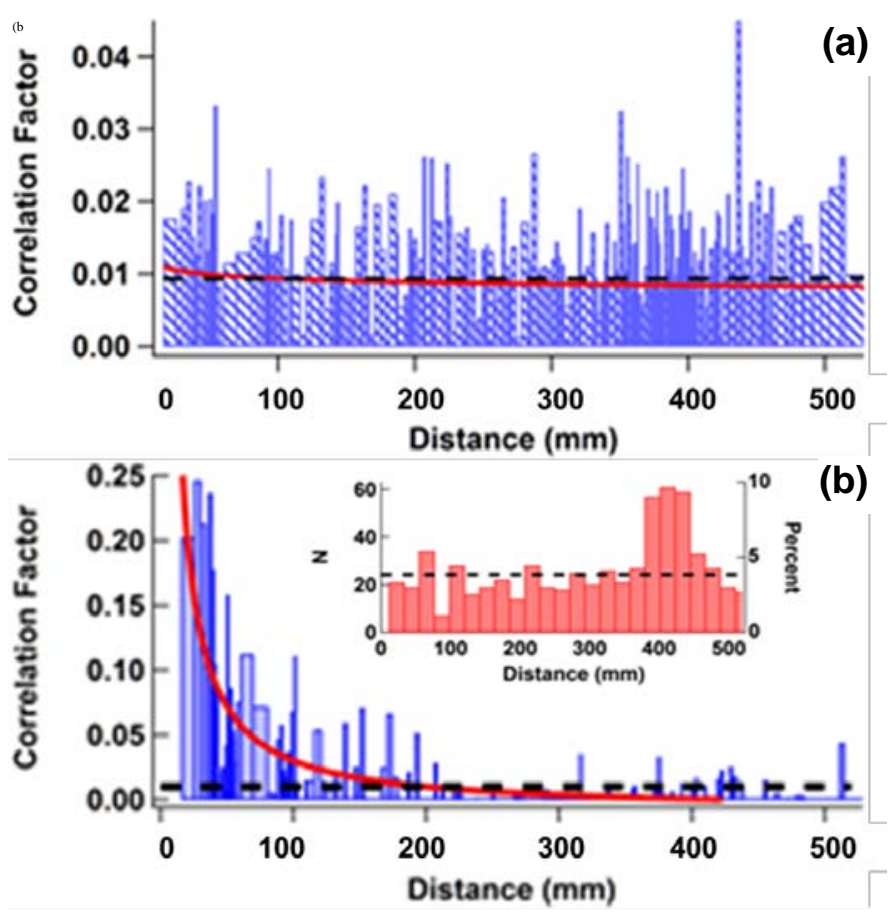

Fig. 7. Correlation factor versus separation distance curves for (a) $25 \mathrm{keV}$ and (b) $40 \mathrm{keV}$ incident energies. The red lines are power law fits based on Eq. (3). The dashed lines show estimated false coincidence factors given by Eq. (4). Inset shows a histogram of separations, $r_{i j}$.

deviations above the average (presumably uncorrelated) correlation rate. Figures 6(a) and 6(b) show the normalized correlation matrices for the two representative energies shown in Figs. 5(a) and 5(b), respectively.

\section{RESULTS}

The possibility that a given arc might stimulate arcs in adjacent "glue dots" was investigated through coincidence correlation analysis. The dependence of such correlations with "glue dot" separation was also studied. The correlation matrix analyses described above were done for incident electron energies between 12 and $40 \mathrm{keV}$ and for fluxes between 0.71 and $5.82 \mathrm{nA}-\mathrm{cm}^{-2}$.

Analyses of the $25 \mathrm{keV}$ data shown in Figs. 5(a) and 6(a), and similar data from other lower energy data sets show very little structure; that is, little to no correlation was observed. We conclude that for lower incident electron energies, most arcs are found to be random events, which occur as localized phenomena when accumulated charge produces an electric field large enough for electrostatic breakdown to occur.

By contrast, for large-incident energy regions, high correlations were seen for groups of nearby "glue dots". Figs. 5(b) and 6(b) are examples of such behavior for a $40 \mathrm{keV}$ incident beam. Higher correlations are most evident amongst the most closely spaced "glue dots" in (i) the lower left corner (dots 16-18 and 33-36) and (ii) between adjacent, peripheral "glue dots" along the matrix first off-diagonal elements. Note the approximate symmetry of the correlation matrix, particularly for these higher correlated "glue dot" pairs confirms that uncertainties inherent in low count rates do not appreciably skew the statistical results.

The correlation of coincidence events versus the distance between samples was also tested. To determine how the 
correlation values scaled with sample separation, these distances, $r_{i j}$, were computed for each sample pair with the Pythagorean theorem using the pixel values of the center of each region. A histogram of the separation distances between "glue dot" pairs is shown in the inset of Fig. 7, with $20 \mathrm{~mm}$ wide separation bins.

The correlation values were graphed versus sample separation, as shown in Fig. 7. A power law of the form

$$
C F(r)=C F_{0} \cdot r^{n}
$$

was fit to these data.

The false coincidence factor for two uncorrelated "glue dots" is given by

$$
C_{i, j}^{u n}=(R)^{2} \cdot \Delta T_{\text {run }} \cdot \Delta t_{\text {frame }},
$$

where $R$ is the average arc rate for a run, $\Delta T_{r u n}$ is the duration of a run, and $\Delta t_{\text {frame }}$ is the duration of a frame. For comparison, the false coincidence factors are 0.009 and 0.010 for the $25 \mathrm{keV}$ and $40 \mathrm{keV}$ data shown in Figs. 7(a) and 7(b), respectively.

Again, little or no correlation was found for lower-incident energy data. For $25 \mathrm{keV}$ data in Fig. 7(a), the fit was nearly independent of distance with $n=0 \pm 2$ and with $C F_{o}=0.01 \pm 0.04$ equal to $C_{i, j}^{u n}$. By contrast, a clear trend was found for some higher-incident energy data sets such as for the $40 \mathrm{keV}$ data shown in Fig. 7(b). A power law fit to these data found an inverse relation between the correlation factor and the separation distance, with a power $n$ of $-1.06 \pm 0.09$ and a coefficient $C F_{o}=(5 \pm 1) \mathrm{mm}$. At the smallest separations of 20$30 \mathrm{~mm}$, the correlation factor is $\sim 25$ times the uncorrelated false coincidence factor.

\section{CONCLUSION}

The results of this study found little to no temporal or spatial correlation was observed between different isolated dielectric "glue dots" for lower incident electron energies; most electroninduced arcs were found to be random events, which occurred as localized phenomena when accumulated charge produced an electric field large enough for electrostatic breakdown to occur. However, for higher incident energies (and consequently, higher power and dose rates), correlation of coincidence arcing between some regions was observed. The coincidence rates of these correlated regions also exhibited a well defined trend with separation distance; closer regions tended to be more correlated.

One possible explanation for the lack of correlated arcing at lower incident electron energies may be the need for the samples to be charged close to their individual breakdown potentials in order for one discharge to trigger other discharges. It appears that coincident arcs are most likely to happen when the incident electron dose rate is large enough to ensure that most of the samples are charged close to their respective breakdown limits at any given time; this was only seen with incident energies of $40 \mathrm{keV}$ in this study. It may be that for lower energies there was not enough charge in the isolated dielectric "glue dot” samples to have a triggered discharge.
A discharge in one "glue dot" may cause a sudden spike in the electric field of neighboring "glue dots" which could trigger premature arcing. Such stimulated arc rates might reasonably be expected to scale with electric field intensity. If confined to a two dimensional surface (i.e., discharged current spreading out on the conductive plate), the field-and hence the correlation rate-would fall off inversely with separation distance. The power law fit to the arc data found for some higher energy data sets is consistent with this $1 / \mathrm{r}$ power drop off model.

This study was an afterthought of the original study for which the data were collected. As such, there were many deficiencies in experimental design which could be accounted for in future investigations. The "glue dots" were not regular shapes and had a modest range of exposed surface areas and volumes; better design would employ more uniform and evenly spaced insulator regions. The "glue dots" had numerous bubbles in them and asperities, which most likely produced higher fields and a higher arc rate. Because the "glue dots" were positioned around the periphery of the conductive sample, there was not a good sampling of all possible distances. A much better experimental design would employ a square grid of epoxy samples, with one or more uniform separation distance(s). It would be better to do this study with a cooled camera to improve the signal-to-noise ratio as much as possible. A more effective way to block stray light from the electron gun filament would be beneficial. Lastly, better electrometer data would allow for better determination of arcs by looking for simultaneous footprints in optical and electric measurements. It would also be better to isolate the current coming from individual epoxy dots so that better information about charge dissipation for each dot could be acquired (potentially allowing us to see arcs in electrometer data). Alternately, surface voltage measurements for each dot could provide similar information.

\section{ACKNOWLEDGEMENT}

We gratefully acknowledge Charles Bowers of NASA Goddard Space Flight Center and Todd Schneider of NASA Marshall Space Flight Center for their help in acquiring the data analysed in this study.

\section{REFERENCES}

[1] Dennison, J., Jensen, A.E., Dekany, J., Wilson, G., Bowers, C.W. \& Meloy, R. (2013). Diverse Electron-induced Optical Emissions from Space Observatory Materials at Low Temperatures, In Proc. Soc. Photo-Optical Instrum. Engineers Cryogenic Optical Systems and Instrum. Conf., Vol. 8863, pp. 88630B1-88630B15.

[2] Andersen, A., Moser, K. \& Dennison, J. (2016). Perspectives on the Distributions of ESD Breakdowns for Spacecraft Charging Applications, Proc. $14^{\text {th }}$ Spacecraft Charging Tech. Conf., Space Research and Technology Centre, European Space Agency (ESA/ESTEC), Noordwijk, The Netherlands.

[3] Leach, R.D. \& Alexander, M.B (1995) Failures and anomalies attributed to spacecraft charging, NASA Reference Publication 1375, NASA Marshall Space Flight Center.

[4] Dekany, J., Christensen, J., Dennison, J.R., Jensen, A.E., Wilson, G., Schneider, T., Bowers, C.W. \& Meloy, R. (2015). Variations in Cathodoluminescent Intensity of Spacecraft Materials Exposed to Energetic Electron Bombardment, IEEE Tran. Plasma Science, 43(11), pp. 3948-3954.

[5] Dekany, J., Johnson, R.H., Wilson, G., Evans, A. \& Dennison, J.R. (2014). Ultrahigh Vacuum Cryostat System for Extended Low Temperature Space Environment Testing, IEEE Trans. on Plasma Sci., 42(1), pp. 266-271. 
[6] Jensen, A.E., Wilson, G., Dekany, J., Sim, A.M. \& Dennison, J.R. (2014). Low Temperature Cathodoluminescence of Space Observatory Materials, IEEE Trans. on Plasma Sci., 42(1), pp. 305-310.

[7] Yacobi, B.G. \& Holt, D. B., (1990) Cathodoluminescence Microscopy of Inorganic Solids, New York, Plenum.

[8] Griseri, V., Dissado, L A, Fothergill, JC, Laurent C and Teyssedre, G, (2001). Photoluminescence, recombination induced luminescence and electroluminescence in epoxy resin, J. Phys. D: Appl. Phys. 34, pp. 5342540.

[9] Wilson, G., Dennison, J.R., Jensen, A.E. \& Dekany, J. (2013). Electron Energy-Dependent Charging Effects of Multilayered Dielectric Materials, IEEE Trans. on Plasma Sci., 41(12), pp. 3536-3544.

[10] Kerwien, C.M., Malandro, D.L., \& Broomall, J. R. (2016), Large Area DC Dielectric Breakdown Voltage Measurement of BOPP and PTFE Thin Films, Proc. 2016 IEEE Conf. on Electrical Insulation and Dielectric Phenomena, pp. 486-489.

[11] Jensen, A.E., Dennison, J.R., Wilson, G. \& Dekany, J. (2013). Nanodielectric Properties of High Conductivity Carbon-Loaded Polyimide under Electron-Beam Irradiation, Proceedings of the 2013 IEEE Intern. Conf. on Solid Dielectrics (ICSD), Bologna, Italy, pp. 730-735.

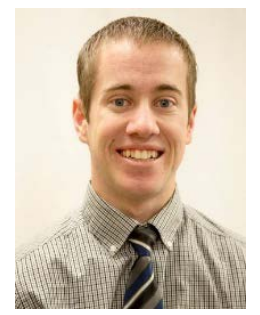

Justin Christensen is currently a graduate student at Utah State University in Logan, UT pursuing an MS in physics. He received a BS degree in physics from USU in 2014. He has worked with the Materials Physics Group for four years His work has focused on electron emission and cathodoluminescent studies related to spacecraft charging.

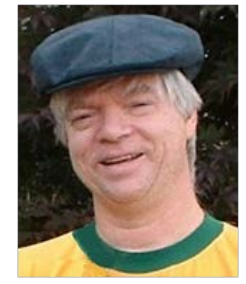

JR Dennison received the B.S. degree in physics from Appalachian State University, Boone, NC, in 1980, and the M.S. and Ph.D. degrees in physics from Virginia Tech, Blacksburg, in 1983 and 1985, respectively. He was a Research Associate with the University of MissouriColumbia before moving to Utah State University (USU), Logan, in 1988. He is currently a Professor of physics at USU, where he leads the Materials Physics Group. He has worked in the area of electron scattering for his entire career and has focused on the electron emission and conductivity of materials related to spacecraft charging for the last two decades. IEEE member.

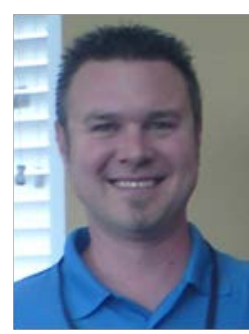

Justin Dekany is currently a graduate student at Utah State University in Logan, UT pursuing an MS in physics. He received a BS degree in physics from USU in 2010. He has worked with the Materials Physics Group for six years on electron transport measurements, electrostatic discharge tests, electron emission measurements, and luminescence studies related to spacecraft charging. 